ry Clinton einerseits den Stellenwert digitaler Medien respektive Plattformen in der öffentlichen Kommunikation und damit einhergehenden Social-Media- oder Plattformlogiken. So sorgten das Thema Microtargeting, also personifizierte, auf Daten und Algorithmen basierte Wahlwerbung, wie auch die Verbreitung von desinformativen Inhalten im politischen Bereich für Diskussion. Es etablierten sich alternative Newssites, darunter besonders präsent Breitbart, die dem republikanischen Präsidentschaftskandidaten zuspielten, dabei wenig Wert auf den Wahrheitsgehalt in ihrer Berichterstattung legten und nachweisbar falsche, desinformative Inhalte verbreiteten (Allcott \& Gentzkow, 2017). Und jüngst machte der Ausbrauch der Corona-Pandemie 2020 deutlich, dass sich insbesondere in krisenhaften Phasen der Raum für alternative Weltdeutungen öffnet. Diese Beispiele belegen, dass national wie auch international in modernen digitalen Gesellschaften von einer Zunahme an Polarisierungstendenzen auszugehen ist. Dies gab Anlass dazu, den deutschsprachigen Raum, der in der aktuellen Forschung diesbezüglich unterrepräsentiert ist, genauer zu analysieren und das Spiel zwischen Öffentlichkeit und Gegenöffentlichkeit zu untersuchen. Der Fokus auf alternative Nachrichtenmedien sollte zudem ein spezifisches Phänomen aufgreifen und deren Bedeutung für den deutschsprachigen Raum ermitteln.

Die sich anschliessenden Subkapitel sind wie folgt gegliedert: Kapitel VIII.1 resümiert die empirischen Befunde und setzt diese mit theoretischen Annahmen in Beziehung. Die wesentlichen Inhalte werden resümiert, wobei die Forschungsfragen reflektiert und zusammenfassend beantwortet werden. Jede Studie weist gewisse Limitationen auf. Diese werden in Kapitel VIII.2 aufgegriffen. In Kapitel VIII.3 erfolgt ein abschliessender Ausblick.

\title{
VIII.1 Gesamtresümee
}

Die forschungsleitende Fragestellung dieser Studie war, welchen Stellenwert deutschsprachige alternative Nachrichtenmedien in der digitalen Öffentlichkeit einnehmen, wie diese zu definieren sind und inwiefern sie untereinander im digitalen Raum vernetzt sind. Hierfür wurde ein multimethodisches Studiendesign gewählt, das sich schwerpunktmässig aus einer qualitativen, interpretativen und phänomenologischen Perspektive dem Untersuchungsgegenstand alternativer Nachrichtenmedien zuwandte.

Forschungsfrage 1 widmete sich insbesondere einer Bestandsaufnahme alternativer Online-Nachrichtenmedien in Deutschland, Österreich und der deutschsprachigen Schweiz. Hierfür wurden in einem ersten Forschungsschritt sämtliche öffentliche Websites induktiv erhoben, die per Selbstverständnis eine alternative Haltung gegenüber der politischen und medialen Öffentlichkeit einnehmen. Dieses Kriterium wurde soweit wie möglich gefasst, um ein möglichst 
breites Sample zu erzielen; Basis dafür bildeten die Selbstbeschreibungen auf den Websites. Zudem wurden nur Websites in das Sample mitaufgenommen, die regelmässig aktuelle Nachrichten verbreiten. Blogs von einzelnen Akteur_innen wurden ausgeschlossen, sofern sie nicht als Nachrichtenmedium auftreten und sich entsprechend selbst definieren. Primäre Suchquelle dieser Websites war die Suchmaschine Google, auf der über unterschiedliche Suchbegriffe (z. B. «alternativ», «unabhängig», «frei», «unzensiert») und Suchbegriff-Konstellationen nach alternativen Medien in Deutschland, Österreich und der deutschsprachigen Schweiz recherchiert wurde. Weiter wurden mit den gleichen Suchbegriffen Google Alerts erstellt, wodurch im Zeitraum Oktober 2018 bis August 2019 sämtliche Google-Treffer automatisch aufgezeichnet wurden. Zusätzlich wurden sämtliche Websites, die den Untersuchungskriterien entsprachen, auf weitere Verlinkungen bezugnehmend auf alternative Nachrichtenseiten untersucht, wodurch das Sample vervollständigt wurde. Daraus resultierte ein bereinigtes Sample von 178 alternativen Newswebsites, für die monatliche Seitenzugriffe via Similarweb wie auch deren Reichweiten auf Facebook, Twitter und YouTube erhoben wurden, sofern die Websites auf diesen Plattformen mit Accounts vertreten sind. Somit war es möglich, einen ersten deskriptiven Überblick über alternative Nachrichtenmedien im DACH-Raum zu geben. Es zeigte sich, dass knapp die Hälfte der untersuchten Websites unter 100'000 monatliche Seitenzugriffe verzeichnete und nur ein kleiner Ausschnitt davon, nämlich 18 Websites, über 1 Million an Seitenzugriffen zählte. Dabei handelt es sich vor allem um Nachrichtenseiten aus Deutschland, wie beispielsweise die Epoch Times Deutschland, Sputnik Deutschland oder Politically Incorrect - Nachrichtenmedien, die durch eine Aufdeckung von Lügen und Geheimnissen des Mainstreams gekennzeichnet sind. Verglichen mit den monatlichen Seitenzugriffen von professionellen Nachrichtenmedien, handelt es sich aber selbst bei diesen Top-Sites um relativ geringe Zugriffszahlen. Über $70 \%$ der analysierten Websites sind zudem mit Accounts auf Facebook und Twitter vertreten, etwa die Hälfte auf YouTube. Top-Accounts auf diesen Plattformen zählen Abonnent_innen-Zahlen im sechsstelligen Bereich, was fallweise mit den Accounts professioneller Nachrichtenmedien vergleichbar ist, wenngleich sie trotzdem geringer ausfallen. Russia Today Deutsch beispielsweise verzeichnet auf Facebook über 380'000 Abonnent_innen, die Süddeutsche Zeitung etwa über 500'000. Die deskriptive Analyse der Newssites zeigte deutlich, dass die theoretisch antizipierte Metapher «Longtail-Öffentlichkeit» auf das Phänomen alternativer Nachrichtenmedien zutrifft. Insofern konnten einzelne alternative Nachrichtenseiten mit beachtlichen Zugriffszahlen identifiziert werden, während der Grossteil davon im «langen Schweif» der Öffentlichkeit verteilt ist.

Nach dieser Bestandsaufnahme erfolgte eine qualitative, interpretative Analyse der Selbstbeschreibungen alternativer Nachrichtenmedien, unter Verwendung der Grounded-Theory-Methodologie (Forschungsfrage 2). Hierbei dienten 
textbasierte und visuelle Daten der Websites als Untersuchungsgrundlage, die Aufschluss über das Selbstverständnis - den subjektiven Sinn alternativer Nachrichtenmedien - gaben. Als Datenbasis fungierte der Pool alternativer Newssites, der im Rahmen der Bestandsaufnahme (FFI) gesampelt wurde. Daraus wurden auf Basis des Theoretical Samplings der Grounded Theory nach und nach Daten in die Analyse miteinbezogen, bis eine theoretische Sättigung erzielt wurde. Insgesamt wurden 56 Websites aus den drei Untersuchungsländern qualitativ analysiert. Neben einer Definition alternativer Nachrichtenmedien ergab sich eine typologische Unterscheidung dieser. Alternative Nachrichtenmedien zeichnen sich demnach durch ihre Positionierung als Vierte Gewalt aus, indem die hegemoniale politische und mediale Öffentlichkeit kritisiert und kontrolliert wird. Dabei agieren sie - je nach Typ - entweder in einer ergänzenden Funktion gegenüber etablierten Medien und versuchen, eine Alternative zum kommerzialisierten Mediensystem einzunehmen. Oder aber sie definieren sich als klare Opposition zum Mainstream. Weiter können alternative Nachrichtenmedien anhand ihrer ökonomischen und sozialen Strukturen wie auch Medieninhalten weiter differenziert werden. Einen Mehrwert für die aktuelle Forschung zum Thema Alternativmedien bietet in diesem Zusammenhang die Unterscheidung in vier Typen alternativer Nachrichtenmedien anhand dieser Definitionskategorien. Typ I, der «Aufdecker der Mainstreamlügen», der im Datenmaterial am häufigsten identifiziert wurde, definiert sich vor allem durch seine Aufdecker-Funktion gegenüber professionellen Nachrichtenmedien. Ziel ist es, die «Fake News» des Mainstreams aufzudecken und der Öffentlichkeit wahrheitsgemässe Berichterstattung zu offerieren. Typ I ist sowohl hinsichtlich sozialer als auch ökonomischer Strukturen hoch professionalisiert - was der Annahme im Forschungsstand widerspricht, dass alternative Medien laienhaft, nicht kommerziell und anti-hierarchisch agieren würden (Atton, 2011). Nachrichtenseiten dieses Typs - vor allem jene, die reichweitenstark agieren ( $\mathrm{FF} 1$ ), - arbeiten teilweise mit professionellen Journalist_innen und Redaktionen, zum Teil aber auch laienhaft (vor allem im hinteren Teil des «Longtails»). Die Seiten sind insofern kommerziell ausgerichtet, als neben Spendenaufrufen häufig auch kostenpflichtige Abonnements angeboten werden oder Werbung auf den Websites integriert ist. Bezogen auf die Medieninhalte fokussieren Medien des Typs I vor allem Kritik am Establishment, Migrationsthemen oder Gender-Kritik. Sie stehen zwar laut Selbstbeschreibung für journalistische Werte wie Unabhängigkeit und Meinungsfreiheit ein, wehren sich aber gleichzeitig gegen Political Correctness. Dies verdeutlicht sich im rhetorischen Stil, der von Ironie und Zynismus gegenüber dem Mainstream geprägt ist. Die relativ hohe Professionalisierung des Typs I zeigt sich auch rein visuell. Insofern ist das Layout von Medien dieses Typs meist ähnlich jenem von professionellen Informationsmedien. Eine «Alternative» wird dadurch nicht deutlich, vielmehr gestaltet es sich für die Nutzer_innen als schwierig, zwischen professionellen 
und alternativen Nachrichtenmedien dieses Typs zu unterscheiden. Typ II, «Verschwörung und Spiritualität», ähnelt Typ I in dem Aspekt, die herrschende Politik und den medialen Mainstream kritisch zu hinterfragen. Ziel ist es, Geheimnisse des Mainstreams aufzudecken. Allerdings wird dabei - im Gegensatz zu Typ I davon ausgegangen, dass hinter gesellschaftlichen Ereignissen eine grössere Elitenverschwörung stecke und nichts auf Zufall beruhe. Infolgedessen behandeln Typ I und II häufig ähnliche Themen, die allerdings unterschiedlich gerahmt werden. Auch bezugnehmend auf die sozialen und ökonomischen Strukturen sind deutliche Unterschiede erkennbar. Typ II agiert wesentlich laienhafter als Typ I, finanziert sich allenfalls über Spendenaufrufe und Inhalte werden vorwiegend von Lai_innen produziert. Medien des Typs II sehen sich selbst als Geheimnislüfter oder Weltverbesserer und setzen sich abgesehen von verschwörungstheoretisch konnotierten Themen mit Esoterik und Spiritualität auseinander. Ihre Alternative zum Mainstream wird auch rein visuell deutlich: Das Layout der Websites ist von einer dunklen Farbgebung und teilweise düsteren Symboliken (z. B. Weltuntergang) gekennzeichnet. Typ III, «Aufstand der Zivilgesellschaft», steht in starkem Kontrast zu den ersten beiden Typen und kann inhaltlich mit der Alternativpresse der neuen sozialen Bewegungen der 1960er- und 1970er-Jahre verglichen werden. Das Selbstverständnis einer Vierten Gewalt gegenüber der vor allem politischen Hegemonie ergibt sich durch die Kritik sozialer Ungleichheit, des Kapitalismus oder Rechtspopulismus. Medien des Typs III verstehen sich als Stimme der Zivilgesellschaft, die soziale Missstände anspricht. Typ III agiert vergleichsweise semi-professionell, sowohl aus sozial- als auch ökonomisch-struktureller Sicht. Medien-Produzent_innen sind teilweise Lai_innen oder Vertreter innen von NGOs; finanziert werden die Medien vorwiegend über Spenden. Typ III widmet sich inhaltlich vor allem sozialistischen Themen (entsprechend häufig findet sich eine rote Farbgestaltung der Websites), mit einem Fokus auf sozialer Gerechtigkeit und Kapitalismuskritik. Aus rhetorischer Sicht sind die Medienbeiträge mobilisierend, im Sinne eines Protestcharakters. Auch Typ IV, «Die seriöse Alternative», unterscheidet sich stark von den restlichen Typen alternativer Nachrichtenmedien. Eine Vierte Gewalt ergibt sich bei diesem Typ vor allem insofern, als die mediale Öffentlichkeit insbesondere aufgrund einer zunehmenden Kommerzialisierung kritisiert und vertiefte Hintergrundberichterstattung eingefordert wird. Typ IV beruft sich auf demokratische Werte und möchte den Leser_innen vertiefte Recherchen bieten, um sich ein Bild von der Gesellschaft machen zu können. Typ IV verzeichnet den vergleichsweise höchsten Professionalisierungsgrad in Bezug auf soziale und ökonomische Strukturen. Oftmals als Start-ups gegründet werden die Medieninhalte hauptsächlich von professionell ausgebildeten Journalist_innen mit redaktioneller Leitung produziert und die Inhalte werden über kostenpflichtige Abonnements zur Verfügung gestellt. Inhaltlich beruft sich Typ IV auf klassische journalistische Werte und Standards und 
steht für fundierten, unabhängigen Journalismus ein. Thematisch fokussiert Typ IV gesellschaftlich relevante Themen, beispielsweise aus Politik und Wirtschaft. Die selbst auferlegte Seriosität verwirklicht sich auch visuell, indem vorwiegend schlichte Designs der Websites gewählt werden.

Die typologische Unterscheidung wird als wesentlich für die Definition alternativer Nachrichtenmedien in digitalen Öffentlichkeiten erachtet, da die bisherige Forschung zumeist einen sehr einschränkenden Blick auf das Phänomen gerichtet hat - so werden vor allem Alternativmedien der Typen I und II fokussiert. Typ III verdeutlicht, dass die Ursprünge alternativer Nachrichtenmedien auch in digitalen Sphären weiterhin Bestand haben. Soziale Bewegungen oder Protestbewegungen machen sich demzufolge den partizipativen Charakter des Internets zunutze, um zu mobilisieren. Die Typen I, II und III spiegeln zudem theoretische Annahmen zum Gegenöffentlichkeitskonzept wider, da bei allen dreien das subjektiv empfundene Eliten-Aussenseiter_innen-Gefälle deutlich wird und sie sich klar gegen die hegemoniale Öffentlichkeit positionieren. Typ IV ist wiederum von einer weniger ausgeprägten oppositionellen Haltung gegenüber dem Mainstream gekennzeichnet. Kritisiert wird allerdings der Wandel des Mediensystems selbst, weshalb die Begrifflichkeit Alternativmedien durchaus gerechtfertigt ist.

Im Zuge von Typenbildungen stellt sich häufig die Frage, inwieweit diese über einen längeren Zeithorizont hinweg übertragbar sind. Unter Bezugnahme auf die Theorie des sozialen Wandels (Imhof, 1996) ist davon auszugehen, dass gesellschaftlicher Wandel insbesondere von Krisenphasen geprägt ist, die neue, alternative Deutungsmuster evozieren. Insofern ist davon auszugehen, dass sich - vor allem in hybriden Mediensystemen (Chadwick, 2017) - weitere Typen alternativer Nachrichtenmedien potentiell ausbilden können. Gleichzeitig zeigt die historische Einordnung von Alternativmedien, dass z. B. die Wurzeln des Typs III, «Aufstand der Zivilgesellschaft», in den 1960er Jahren und den neuen sozialen Bewegungen liegen, was für eine Beständigkeit dieses Typs spricht. Auch stieg die Bedeutung von desinformativen und verschwörungstheoretischen Medien (Typ I, II) in den vergangenen Jahren. Vor allem die aktuelle globale Corona-Pandemie zeigt, dass einschlägige Stimmen in Krisenphasen lauter werden und nicht von kurzlebiger Dauer sind. Typ IV, der aus quantitativer Sicht im Untersuchungszeitraum eher selten vertreten war, verdeutlicht zudem, dass in digitalen Öffentlichkeiten auch der Raum für professionell journalistische Nachrichtenangebote in Form von Medien-Startups abseits des «Mainstreams» geöffnet wurde.

Die typologische Konzeptualisierung alternativer Nachrichtenmedien war prägend für die weiteren methodischen Schritte der vorliegenden Arbeit. Vor allem aus dem Forschungsstand, aber auch aus den bisherigen analytischen Schritten wurde die These abgeleitet, dass alternative Nachrichtenmedien durch einen starken Vernetzungscharakter geprägt sind. So gehen Theorien zur digitalen Transformation der Öffentlichkeit davon aus, dass die Vernetzung eine wesent- 
liche Logik von Digitalplattformen ist (z. B. Castells, 2010 [1996]; Eisenegger, 2021; Klinger \& Svensson, 2015; van Dijck \& Poell, 2013). Auch aktuelle Gegenöffentlichkeitskonzepte beschreiben das Potential für Gegenöffentlichkeit, sich im Internet stärker miteinander zu vernetzen und sich gegenseitig zu mobilisieren (z. B. Fenton \& Downey, 2003; Kaiser \& Rauchfleisch, 2019). Weiter wurde in der theoretischen Auseinandersetzung dieser Arbeit deutlich, dass Öffentlichkeit und Gegenöffentlichkeit relational zueinanderstehen, weshalb es wesentlich erscheint, diese Relationen genauer zu betrachten. In diesem Zusammenhang wurde versucht, die Netzwerkstrukturen von alternativen Medien auf der Digitalplattform Twitter zu untersuchen, um erstens herauszudestillieren, wie alternative Medien untereinander über Follower_innen und wechselseitige Retweets vernetzt sind, aber auch in Relation zu professionellen Nachrichtenmedien und weiteren Akteur_innen in der Twitter-Öffentlichkeit stehen (Forschungsfrage 3). Twitter eignete sich deshalb als empirischer Untersuchungsausschnitt, da die Plattform erstens stark von journalistischen wie auch politischen Akteur_innen genutzt wird und zweitens die Bestandsaufnahme alternativer Nachrichtenmedien im DACH-Raum (FF1) deutlich zeigte, dass Twitter eine häufig genutzte Plattform von alternativen Nachrichtenmedien darstellt. Die Datenerhebung basierte auf dem Sample alternativer Newswebsites wie auch induktiv erhobener URLs professioneller Nachrichtenmedien im DACH-Raum. Diese URLs dienten als Suchbegriffe für die Datenerhebung unter Verwendung von R und mittels Verknüpfung mit der Twitter-API. Der Erhebungszeitraum erstreckte sich vom 15. September 2019 bis zum 27. Oktober 2019 während dreier politischer Wahlen in den Untersuchungsländern. Sämtliche Tweets, in die jene URLs eingebettet wurden, waren Teil des resultierenden Samples. Es wurden zwei Datensätze (alternative Nachrichtenmedien exklusiv; alternative und professionelle Nachrichtenmedien gesamt) erstellt, bereinigt und netzwerkanalytisch - unter Verwendung von R und Gephi - untersucht. Das Follower-Netzwerk alternativer Nachrichtenmedien umfasst drei Communities, die stark miteinander verschränkt sind: die Community Schweizer alternativer und professioneller Nachrichtenmedien, die Community alternativer Nachrichtenmedien des Typs IV, professioneller Nachrichtenmedien und politisch eher links orientierter Akteur_innen, und schliesslich die Community der Alternativmedientypen I und II und rechtspopulistischer Akteur_innen. Ein ähnliches, aber differenzierteres Bild ergab sich im Follower-Netzwerk unter Einschluss professioneller Nachrichtenmedien. Deutlich wurde hier vor allem, dass unterschiedliche Ländercommunities existieren. Innerhalb dieser sammeln sich professionelle Nachrichtenmedien aus dem jeweiligen Land, aber auch alternative Nachrichtenmedien der Typen III und IV entsprechend ihrer Länderzuordnung. Besonders interessant ist der Befund, dass die Community alternativer Nachrichtenmedien der Typen I und II länderübergreifend existiert und weitere rechtspopulistische, internationale Akteur_innen beinhaltet. Dies verdeutlicht, dass vor allem 
rechtspopulistische Akteur_innen und alternative Nachrichtenmedien mit einer diesbezüglich zustimmenden Haltung länderübergreifend mobilisieren und entsprechend auch ein internationales Publikum bedienen. Die Retweet-Netzwerkstrukturen gaben Aufschluss über die Relationen zwischen Akteur_innen in Form von Retweets, also des Teilens von Beiträgen. Diese sind vor allem deshalb von Interesse, da diese Strukturen offenbaren, welche Akteur_innen sich gegenseitig die Bälle zuspielen und ggf. dadurch ihre gemeinsame Deutungsmacht erhöhen. Das Retweet-Netzwerk alternativer Nachrichtenmedien verdeutlichte die wesentliche (und somit bestätigte) Unterscheidung in vier Typen wie auch die Annahme, dass Relationen vor allem unter Medien eines Typs existieren. Im Gegensatz zum Follower-Netzwerk konnten hier stärker voneinander getrennte Communities identifiziert werden. Die Communities können vor allem basierend auf ihrer politischen Ausrichtung voneinander unterschieden werden - besonders gross ist dabei die Differenz zwischen der Community des Typs I und der des Typs IV. Typ II nimmt eine verbindende Funktion zwischen den Communities ein, was nahelegt, dass verschwörungstheoretische Narrative nicht unbedingt von einer politischen Zugehörigkeit abhängen. Nachrichtenmedien des Typs III kommt per se ein geringer Stellenwert in den Netzwerken zu, da sie quantitativ am seltensten auftreten. Ein ähnliches Bild ergab sich im Retweet-Netzwerk alternativer und professioneller Nachrichtenmedien. Die Communities sind in ähnlicher Weise voneinander getrennt, allerdings vermittelt über professionelle Nachrichtenmedien aus den drei Untersuchungsländern. Die Netzwerke gaben Aufschluss über die Strukturen alternativer (und auch professioneller) Nachrichtenmedien. Deutlich zeigte sich, dass per Definition nicht von abgeschlossenen «Echokammern» gesprochen werden kann; vielmehr scheinen (wie angenommen) Verbindungen zwischen Öffentlichkeit und Gegenöffentlichkeit zu existieren.

Forschungsfrage 4 beschäftigte sich mit den Sinnstrukturen zwischen diesen Relationen. Massgeblich dafür waren die Annahmen der relationalen Soziologie, wonach Netzwerksbeziehungen nicht ausschliesslich auf starren Strukturen basieren, sondern vielmehr auf Narrativen zwischen Akteur_innen (vgl. für einen Überblick Emirbayer, 1997). Dieser Logik folgend wurden Tweets mit relationalen Bezugnahmen zwischen Akteur_innen qualitativ inhaltsanalytisch untersucht. Diesbezüglich wurde ersichtlich, dass neben klassischen Medienlogiken auch Plattformlogiken (z. B. durch die Verwendung von Plattformfunktionen wie Mentions oder Retweets) einen wesentlichen Stellenwert in Bezug auf die Popularität von Beiträgen einnehmen, im Sinne einer Anreicherung an Kapital (Bourdieu, 2013 [1979]). Wie im Rahmen der Netzwerkanalysen belegt werden konnte, agieren gleichgesinnte Akteur_innen in homophilen Gruppen respektive sozialen Kreisen. Als wesentlich ist dahingehend die Ingroup- und Outgroup-Dynamik $\mathrm{zu}$ nennen. Obwohl netzwerkanalytisch Relationen zwischen unterschiedlichen Communities alternativer und professioneller Nachrichtenmedien identifiziert 
werden konnten, hat die qualitative Analyse ergeben, dass die jeweiligen Bezugnahmen unterschiedlichen Logiken folgen. So sind Relationen innerhalb der Communities im Sinne eines Vergemeinschaftungsprozesses (Weber, 1985 [1922]) stark positiv konnotiert und verfolgen das Ziel, ein Gefühl der Zugehörigkeit zu schaffen - und schliesslich die Reichweiten Gleichgesinnter zu erhöhen. Oppositionelle Gruppen, wie etwa Mainstreammedien (oder: die «Elite»), werden fallweise stark negativ bewertet oder gar diffamiert, vor allem von Typ I und Typ II alternativer Nachrichtenmedien. Medien dieser Typen zelebrieren ihre Aussenseiter-Rolle gegenüber der Elite (Elias \& Scotson, 2002 [1965]) und vernetzen sich mit gleichgesinnten Akteur_innen z. B. aus Politik und Wissenschaft. Wesentlich für positive Bezugnahmen ist die Bestätigung der eigenen Narrative, die gleichzeitig die Basis dafür darstellt, welche Expert_innen verlinkt respektive zitiert werden. Die Relationen zwischen Öffentlichkeit und Gegenöffentlichkeit äusserten sich je nach Alternativmedientyp sehr unterschiedlich. Vor allem Typ I und II nehmen eine ablehnende Haltung gegenüber dem Mainstream ein. Verlinkungen in Form von Retweets oder Mentions werden vor allem dazu genutzt, die eigene Gruppenzugehörigkeit herauszustreichen. Positive Bezugnahmen finden nur dann statt, wenn einzelne Beiträge der Mainstreammedien die Narrative der Gruppe stützen, was teilweise in der Berichterstattung von Boulevardmedien der Fall ist. Typ III, der gesamthaft betrachtet am seltensten in der Twitter-Öffentlichkeit aufzufinden war, nimmt in erster Linie auf den politischen Mainstream Bezug. Entsprechend der Ausrichtung der Medien dieses Typs finden sich positive Bezugnahmen gegenüber sozialdemokratischen Parteien und Politiker_innen, während Verlinkungen rechtskonservativer Akteur_innen mit deutlicher Kritik einhergehen. Typ IV, der sich vor allem auf demokratische Werte beruft, nimmt die vergleichsweise geringste oppositionelle Haltung gegenüber dem Mainstream ein, was sich auch in den veröffentlichten Beiträgen widerspiegelt. Im Sinne einer Vierten Gewalt weisen Medien dieses Typs zwar auf fehlerhafte Berichterstattung hin, tendenziell ist die Grundhaltung gegenüber professionellen Nachrichtenmedien allerdings positiv. Insofern ist es auch nicht verwunderlich, dass Mainstreammedien - wenn sie überhaupt auf alternative Nachrichtenmedien Bezug nehmen - vor allem auf diesen Typ IV verweisen. Dies kann auch damit zusammenhängen, dass vermieden wird, alternativen, diffamierenden Stimmen eine Bühne zu geben. Der individuelle Habitus (Bourdieu, 2013 [1979]) der unterschiedlichen Alternativmedientypen zeigte sich insbesondere in der gewählten Rhetorik. Es wurde deutlich, dass alternative Medien der Typen I und II stark wertend und teilweise sogar diskreditierend gegenüber bestimmten Gruppen (nicht nur politischen und medialen Eliten, sondern z. B. auch Migrant_innen oder Klimaschützer_innen) argumentieren. Typ III verdeutlicht zwar seine politische Position, bleibt dabei aber vergleichsweise sachlich. Vor allem Typ IV drückt seine professionelle Ausrichtung über einen gewählten und sachlichen Diskursstil aus. Obwohl bei allen vier 
Typen Relationen zum Mainstream identifiziert wurden, liess sich doch erkennen, dass dies nicht mit einer argumentbasierten Debatte zwischen unterschiedlichen Gruppen einhergeht. Vielmehr manifestieren sich polarisierte Haltungen umso mehr, wenn zwar auf oppositionelle Gruppen verwiesen wird, dies allerdings in einem diffamierenden, nicht auf Vernunft basierenden Ton geschieht.

Gesamthaft betrachtet konnten durch die unterschiedlichen Forschungsschritte und -methoden - vor allem in ihrer Kombination - aufschlussreiche Erkenntnisse erzielt werden. Zunächst konnte das digitale Feld alternativer Nachrichtenmedien im DACH-Raum induktiv aufgearbeitet werden, obschon dies nur einen empirischen Ausschnitt darstellen kann. Die typologische Unterscheidung alternativer Nachrichtenmedien ist insofern - auch für weitere Forschungen in diesem Themenbereich - wesentlich, als sie den Blick für unterschiedliche alternative Nachrichtenangebote im Internet öffnet. Obgleich Typ I, der «Aufdecker der Mainstreamlügen», im analysierten Material am präsentesten erschien, wäre es zu kurz gegriffen, Alternativmedien auf diesen Typ - der vor allem im Zusammenhang mit Rechtspopulismus und Desinformation konnotiert ist - definitorisch zu beschränken. Wie auch aus historischer Sicht Gegenöffentlichkeiten in unterschiedlicher Form in Erscheinung treten können, ist dies ebenso - oder vielleicht sogar in noch ausgeprägterer Weise - in digitalen Öffentlichkeiten der Fall. Insofern zeigte sich, dass Alternativmedien nicht per se dysfunktional für die demokratische Öffentlichkeit sind, sondern fallweise auch in ausgeprägterer Weise eine wesentliche Funktion öffentlicher Kommunikation einnehmen können - so vor allem Typ IV, «Die seriöse Alternative». Im Rahmen der netzwerkanalytischen Betrachtung wurde einerseits offensichtlich, dass die typologische Unterscheidung valide ist, da sich ebendiese Typen in den Communities widerspiegelten. Hervorzuheben ist indes, dass es sich um keine abgeschlossenen Teilöffentlichkeiten handelt, da durchaus Bezugnahmen auf öffentliche Gruppen, wie beispielsweise die Mainstreammedien, existieren. Als besonders starke Knoten, im Sinne deutungsmächtiger Akteur_innen, erwiesen sich vor allem einzelne Nachrichtenmedien aus Deutschland, die Typ I zugeordnet wurden. Aber auch Alternativmedien am entgegengesetzten Pol dieses Kontinuums alternativer Medien zeigten durchaus hohe Eingangsgrade, so beispielsweise Media-Watchdogs, die zum Typ IV gezählt werden können. Die interpretative Analyse dieser Relationen kam allerdings vorwiegend zu dem Ergebnis, dass Bezugnahmen zwischen unterschiedlichen Gruppen vor allem dazu dienen, das eigene Gruppenzugehörigkeitsgefühl zu stärken und oppositionelle Haltungen deutlich zu machen. Polarisierende Tendenzen zwischen Öffentlichkeit und Gegenöffentlichkeit wurden so fallweise sehr deutlich. 